\title{
Sorge im Zentrum: Die Folgen der Corona-Krise in Lateinamerika aus Geschlechterperspektive
}

\section{Caroline Kim}

„Die Pandemie hat den Topf geoffnet, in dem seit Langem Ungleichheiten, Ungerechtigkeiten und Asymmetrien brodeln, die die Welt mit Gewalt ordnen."1

(Colectivo Mapeos Feministas 2021: 38)

Seit Anfang 2020 beherrscht die Corona-Krise das Leben der Menschen weltweit. Sie hat nicht nur das komplette Alltagsleben verändert, sondern auch die Schwäche des kapitalistischen Systems offenbart. Die costa-ricanische Anthropologin Montserrat Sagot argumentiert, dass die Corona-Krise mit Blick auf Lateinamerika mehr ist als eine bloße Gesundheitskrise. Sie spricht stattdessen von einer „zivilisatorischen Krise“ mit Auswirkungen auf alle Bereiche des menschlichen Lebens (Montserrat 2020: 02:40). Auch internationale Organisationen wie die Frauenorganisation der Vereinten Nationen, UN Women, bezeichnen die Covid-19 Pandemie als einer der schlimmsten gesundheitlichen, wirtschaftlichen, sozialen und humanitären Krisen in der Region Lateinamerika und Karibik in diesem Jahrhundert (Naciones Unidas 2020: 8).

Dabei ist die Corona-Krise nicht jener Ausnahmezustand, als welcher er oft dargestellt wird. Vielmehr wird durch die Pandemie die Krise des Alltags sichtbar: „Wir leben“, wie die italienische Philosophin Silvia Federici sagt, in einer „permanenten Krise“, einer „alltäglichen Krise des Lebens" (Federici 2020: 19:37). Die bestehenden Ungleichheiten wurden auf dramatische Weise ins Licht gerückt, die Corona-Krise ist eine „Röntgenaufnahme aller Probleme unserer Gesellschaft" (Federici 2020: 01:49:25). Die Notlage, die seit Anfang 2020 noch deutlicher geworden ist - die Prekarität der Arbeit, die kollabierten Gesundheitssysteme, die explodierte Sorgelast, die implodierten Haushalte - ist Teil der Logik eines Systems, das einerseits Wirtschaft und Profit in den Vordergrund stellt und andererseits das Leben strukturell abwertet. La vida, das Leben, wird hier als ein Konzept gefasst, das (nicht nur) lateinamerikanische Feminist"innen seit

1 In diesem Beitrag werden für spanisch- und englischsprachige Originalquellen eigene Übersetzungen verwendet. 
Langem ins Zentrum stellen und für das sie im Konflikt „Kapital vs. Leben" mit kollektiven Alternativen zur kapitalistischen Inwertsetzungslogik streiten.

Dieser Beitrag soll einen Einblick in die feministischen Debatten um die Corona-Krise in Lateinamerika geben und dabei die Folgen aus Geschlechterperspektive in den Blick nehmen. Die Debatten zeigen, wie sehr die aktuelle Krise mit der Krise der Sorgearbeit und mit der geschlechtlichen Arbeitsteilung als einem der Grundpfeiler der geschlechtlichen Ungleichheit verbunden ist. An diesem Grundpfeiler anzusetzen ist aus feministischer Analyse unabdingbar, um Ungleichheit im Zusammenhang mit der Krise und darüber hinaus anzugreifen. Daher sollen schließlich Ideen und Praxen rund um die kollektive Reorganisation von Sorge aufgezeigt werden, die als soziale Transformationsstrategie Zukunftspotenzial haben.

\section{Feministische Perspektiven auf Leben und Sorge}

Viele der Themen, die seit Langem feministische Debatten bestimmen, sind in der Corona-Krise neu beleuchtet worden: das Zuhause (welches Zuhause?), familiäre Beziehungen (was ist Familie?), Gemeinschaft (wie konstruieren wir sie?), soziale Reproduktion (welche Tätigkeiten sind wirklich systemrelevant und wie definieren wir Arbeit?), geschlechtliche Arbeitsteilung (wer erfüllt diese Aufgaben?) und das Leben selbst (welches Leben ist es wert, geschützt zu werden?).

Das Virus hat die tödlichen Mechanismen des Neoliberalismus klarer gemacht: Jahrzehnte der Kürzungen im öffentlichen Gesundheitssektor, Privatisierungen und Externalisierung der gesundheitlichen Dienste, systematischer Abbau und Merkantilisierung sozialer Infrastruktur sind verantwortlich für das kollabierte Gesundheitssystem und die hohen Todeszahlen durch das Coronavirus in Lateinamerika. Damit im Zusammenhang steht die Überausbeutung von prekärer und migrantischer Arbeit, der Abbau von Arbeitsrechten, die Krise des Wohnraums und die explodierende Verschuldung der Haushalte. Das Bild des „despojos“, einer geplünderten öffentlichen Infrastruktur, einem verwüsteten Feld, in dem die Bedingungen zum Überleben von eigenen Ressourcen abhängen, scheint geeignet für diesen Zustand. Die argentinischen Soziologinnen Verónica Gago und Lucía Cavallero schreiben, dass jeder einzelne Kampf gegen Prekarität ein Kampf in der großen „Schlacht des Kapitals gegen das Leben“ (Gago/Cavallero 2020) ist. Die analytische Idee des Konflikts Kapital-Leben erklärt die Ordnung des aktuellen sozio-ökonomischen Systems, das verschiedene Leben entlang intersektionaler Achsen hierarchisiert. Zur Erfüllung der 
Interessen einiger privilegierter Leben werden die Leben anderer, die Erde und ihre Ressourcen geplündert und die kollektive Reproduktion in private Formen der „Produktion” von Kapital verwandelt (vgl. Colectiva XXK 2020: 24). Der Konflikt Kapital-Leben trägt sich auf verschiedenen Territorien aus (die z.B. Körper, Wohnraum, Ernährung oder Sorgearbeit sein können), in denen bestimmte Leben - vor allem die Leben von Menschen im Globalen Süden - gezielt vom Kapital angegriffen werden.

\section{Das (nicht) diskriminierende Virus: Folgen der Pandemie für Menschen in} radikal ungleichen Verhältnissen

"Jahrzehnte des Neoliberalismus explodieren in den immer gleichen Körpern" (Colectivo Mapeos Feministas 2021: 37) heißt es im Dossier zu "Sorgearbeit in der Krise" des Instituto Tricontinental de Investigación Social, einem sozialwissenschaftlichen Institut, das sich einer bewegungsorientierten Forschung in Afrika, Lateinamerika und Asien widmet. Die Autor*innen des Dossiers beziehen sich vor allem auf feminisierte, queere, migrantisierte, rassifizierte und arme Körper, für die die Auswirkungen der Krise am deutlichsten zu spüren sind. Denn die Krise betrifft alle, aber nicht alle gleich. Das Virus selbst diskriminiert nicht, schreibt die deutsche Philosophin Eva von Redecker. Dass menschliche Verletzbarkeit und Sterblichkeit überhaupt wieder als geteiltes Schicksal erfahren worden sind, war ein „Ereignis“, aber diese Verletzbarkeit werde kaum mehr als nur für einen flüchtigen Moment geteilt. Als sozialer Prozess der Ansteckung diskriminiert das Virus sehr wohl (vgl. von Redecker 2020: 150). Welche Leben letztlich betroffen sind, ist auf radikale Weise ungleich, je nachdem, an welchem Ort der Welt und in welcher sozialen Position sie sich befinden. Denn Covid-19 trifft dort am stärksten, wo es am meisten Armut und kein sauberes Wasser gibt, wo die Menschen eng zusammenwohnen und arbeiten. Auch die Fähigkeit auf die Krise und das Virus zu reagieren ist nicht die gleiche für alle, wie die politischen Maßnahmen zur Eindämmung der Pandemie zeigen: Wenn gleiche Maßnahmen in einer ungleichen gesellschaftlichen Realität angewendet werden, werden soziale Ungleichheiten vervielfacht (vgl. Orozco 2020: 7:15). Eine Ausgangssperre für alle hat ungleiche Auswirkungen abhängig von den Lebensbedingungen der Menschen. Nicht alle haben ein Dach über dem Kopf, nicht für alle ist das Zuhause sicher. Der Aufwand die Maßnahmen zur sozialen Isolation einzuhalten, ist unterschiedlich hoch. Entscheidend ist, ob sie trotzdem ein Einkommen haben, wenn sie zuhause bleiben, welche Geschlechterrollen in den Haushalten wirken, welcher Gewalt Menschen in 
ihrem Zuhause ausgesetzt sind und ob das Zuhause zugleich zur Schule und zum Büro, zum Isolierungsort oder aufgrund häuslicher Gewalt zum Gefängnis wird.

Soziale Ungleichheiten waren schon vor der Krise extrem - mit der Krise potenzieren sie sich entlang bekannter Kategorien wie Geschlecht, race und Klasse. Laut Schätzungen der UN-Wirtschaftskommission für Lateinamerika und die Karibik CEPAL ist die Zahl der von Armut betroffenen Menschen in Lateinamerika und der Karibik von 2019 auf 2020 auf 209 Millionen Menschen (ein Plus von 22 Millionen) angestiegen. Das ist ein Niveau, das es nach Jahrzehnten der Armutsbekämpfung zuletzt vor 20 Jahren gegeben hat (CEPAL 2021a: 72f.). Die Zahl der Menschen in einer ernsthaften Hungersituation ist um 269 Prozent angestiegen. Zur gleichen Zeit hat sich die Zahl der Multimilliardär*innen in der Region um 31 Personen erhöht. Die nun 107 reichsten Menschen der Region haben ihren Besitz während des ersten Krisenjahrs noch um 40 Prozent steigern können, ein Vermögen, das in etwa der Wirtschaftsleistung von Ecuador entspricht (vgl. López-Calva 2021).

\section{Sorgearbeit: Die Infrastruktur, die das kollektive Leben aufrechterhält}

Die Corona-Krise hat die Grenzen des Kapitalismus aufgezeigt. Der Markt, der vermeintlich alles regelt, ist nicht imstande gewesen globale Antworten auf die globale Krise zu geben. Die zur Überwindung der Krise systemrelevante Infrastruktur wird insgesamt nur durch feminisierte, prekarisierte und migrantische Arbeit un- oder unterbezahlt aufrechterhalten. Wie keine andere Krise zuvor, ist diese Krise mit der Krise sozialer Reproduktion und Sorgearbeit verwoben. Der explodierte Gesundheitsnotstand zeigt die Notwendigkeit von Sorge für die Gesellschaft: Die gesellschaftliche Abhängigkeit von Sorge und ihre Relevanz für das Funktionieren des Systems ist deutlicher denn je geworden. Die alltägliche Reproduktion des Lebens ist zudem die Infrastruktur, die das kollektive Leben aufrechterhält (vgl. Gago/Cavallero 2020). Hierzu zählt all das, was getan werden muss, um das (soziale) Leben jeden Tag neu zu ermöglichen: sorgen, betreuen, erziehen, pflegen, ernähren, kochen, waschen, putzen, organisieren, haushalten, trösten, lieben, lösen. Die alltägliche Reproduktion ist daher der Bereich, in dem die Krise am intensivsten gelebt wird und der von entscheidender Bedeutung für eine gesellschaftliche Transformation ist, vor allem für Frauen (vgl. Federici 2020: 26:36).

Die reproduktiven Aktivitäten sind die, die auch während der Krise nicht gestoppt werden können, weil sie mit den Grundbedürfnissen des 
menschlichen Überlebens zu tun haben. Dabei gibt es eine naturalisierte, d.h. eine generelle, am biologischen Geschlecht festgemachte Anforderung, Sorgetätigkeiten zu übernehmen, verbunden mit einer vermeintlichen „Opferbereitschaft“, beispielsweise des Mutterseins. Diese Aufgaben werden nicht entlohnt, sind unsichtbar und zugleich systemrelevant. Sie erfordern Anerkennung und sind grundlegend, um die Reproduktion des Lebens zu ermöglichen, also das Leben zu erhalten. Und nicht nur das Leben selbst, auch die kapitalistische Produktion und der Markt sind abhängig von einer konstanten Arbeit der Reproduktion, die nie endet und die zu einem Großteil von Frauen verrichtet wird.

Feministische Bewegungen in Lateinamerika und weltweit blicken auf einen langen Kampf für die Anerkennung der reproduktiven Tätigkeiten zurück, in dem sie die Politisierung der Sorgearbeit vorangetrieben haben. Für die Analyse der Corona-Krise aus feministischer Sicht ist die Einsicht über die Verwundbarkeit des menschlichen Lebens und die gegenseitige Abhängigkeit voneinander als „Grundbedingung unserer Existenz" (Colectiva XXK: 18) zentral. So sind alle Menschen zeitweise Sorgende und von Sorge Abhängige. Sie brauchen sich gegenseitig, sie brauchen Gemeinwohl und soziale Dienstleistungen. Leben wird erst durch das Geben und Erhalten von Fürsorge, Arbeit, Zeit, Wissen, Zuneigung, etc. möglich (Colectiva XXK: 18).

\section{Sorgearbeit in Lateinamerika: Feminisiert und familiarisiert}

Die Ungleichheiten, die aus der geschlechtlichen Arbeitsteilung und aus der gesellschaftlichen Nicht-Anerkennung von Sorgearbeit als geteilte Verantwortung resultieren, vertiefen sich mit der Corona-Krise. Es wird deutlich, welche ungleichen Auswirkungen die aktuelle Krise auf die Leben von Frauen, trans und inter Personen hat, die den überwiegenden Teil der bezahlten und unbezahlten Sorgearbeit schultern. ${ }^{2}$ Diese ungleich ver-

2 Die in diesem Beitrag zitierten Zahlen verschiedener Datenerhebungen unterscheiden zwischen zwei Geschlechtern: Männern und Frauen. Zahlen für nicht-binäre oder inter* Personen tauchen nicht in den Statistiken auf. Oftmals übernehmen aber Menschen außerhalb der binären Geschlechterordnung ähnliche Aufgaben und Rollen, die Frauen zugeschrieben werden. In der lateinamerikanischen Literatur wird oft von „feminisierten Körpern“ gesprochen, als Körper von Menschen, die nach den geltenden Normen als Frauen wahrgenommen werden, die aber ihre geschlechtliche Identität nicht als weiblich definieren. Sie sind vielleicht keine Frauen, ihre Körper werden aber als weiblich gelesen und haben einen ähnlichen 
teilte Sorgelast hat sich während der Corona-Krise noch erhöht. Zu den gewohnten Tätigkeiten kamen durch die Schließung der Bildungseinrichtungen zusätzliche Betreuungs- und Erziehungsaufgaben wie Home Schooling hinzu, die wieder überwiegend von Frauen übernommen wurden (vgl. Batthyány 2020: 24:00-26:06): In Uruguay z.B. gehen bei einer Befragung von UN Women im Jahr 202073 Prozent der Befragten davon aus, dass Mütter für die Betreuung der Schulkinder zuständig sind, im Gegensatz zu nur zehn Prozent, die Väter in die Verantwortung nehmen. Die nicht bezahlten Arbeitsstunden von Frauen stiegen in der Pandemie von 6,9 auf 8,1 Stunden täglich (Männer 3,9 auf 4,6 Stunden). In Chile haben Frauen während der Pandemie im Durchschnitt 5,4 Wochenstunden extra für Schulaufgaben ihrer Kinder aufgewandt, Männer im gleichen Zeitraum 2,4 Stunden die Woche (vgl. CEPAL 2021a: 206f.). Die Naturalisierung von Frauen als sorgende und pflegende Personen, die mit ihren Körpern automatisch und eher unbeachtet die Krise bewältigen, kommt in vorherrschenden Geschlechterrollen und heteropatriarchalen Familienstrukturen zum Tragen, die in der Krise reaktiviert werden.

Im Rahmen der Corona-Krise hat es mitunter eine soziale (und moralische) Wertschätzung für sorgende und pflegende Tätigkeiten gegeben, z.B. für Pflegepersonal. Auch das Wissen um die Abhängigkeit von Sorge hat Eingang in einen Common Sense gefunden. Materielle Konsequenzen dieser Anerkennung sind jedoch überall ausgeblieben. Verónica Gago plädiert daher dafür, dass dieser gesellschaftliche Moment der „Hypervisibilisierung und Überausbeutung" (Gago 2020: 09:45) von Frauen und feminisierten Körpern dafür genutzt wird, dass diese einen materiellen Ausgleich für die investierte Zeit und Arbeit für Reproduktionsaufgaben bekommen. Die Forderung lautet daher, dass symbolische Anerkennung mit Rechten und Entlohnung für diese Arbeit verbunden werden muss.

Die Arbeitsgruppe Sorgearbeit und Geschlecht (Grupo de Trabajo Cuidados y Género) des Zusammenschlusses der lateinamerikanischen Sozialwissenschaften (Consejo Latinoamericano de Ciencias Sociales, CLACSO) hat mehrfach betont, dass sich Sorgearbeit in Lateinamerika dadurch auszeichne, dass sie feminisiert und familiarisiert ist. Die hauptsächliche Sorgelast

sozialen Status wie Frauen innerhalb der binären Geschlechternorm, d.h. oft erleben auch sie eine ähnliche Ungleichbehandlung. Die (oft abwertenden) Eigenschaften und unbezahlten Aufgaben der Sorgearbeit, die gesellschaftlich mit Frauenrollen verbunden werden oder ihnen zufallen, kommen auch bei feminisierten Körpern zum Tragen. In der Analyse der Zahlen ziehe ich Frauen, trans und inter* Personen, feminisierte Körper und queere Menschen mit ein, die zitierten Zahlen beziehen sich mangels Daten nur auf cis-Frauen und cis-Männer. 
fällt auf pflegende Angehörige, die zu einem Großteil Frauen sind. Die geschlechtliche Arbeitsteilung führt zu einer ungleichen Verteilung der Verwendung von Zeit zwischen Frauen und Männern: Bereits vor der Pandemie widmeten Frauen in Lateinamerika und der Karibik zwischen 26 und 40 Wochenstunden der Sorge- und Hausarbeit, eine komplette „Vollzeitstelle“ und durchschnittlich drei Mal mehr Zeit, als Männer denselben Tätigkeiten widmen. Nur 27 Prozent der gesamten auf unbezahlte Sorgearbeit verwendeten Zeit wird von Männern übernommen. Im internationalen Vergleich liegt die Region damit deutlich zurück, in den USA sind es 38, in Schweden 44 und in China 39 Prozent (Batthyány 2020: 04:40-05:15). Bereits vor der Pandemie (2018) konnten laut Zahlen der Internationalen Arbeitsorganisation (ILO) im regionalen Durchschnitt 42 Prozent der Frauen im arbeitsfähigen Alter aufgrund von familiären Sorgeverpflichtungen keiner Lohnarbeit nachgehen, im Gegensatz zu nur fünf Prozent der Männer (Batthyány 2021: 14:40). Obwohl Sorgearbeit ein struktureller Knotenpunkt der geschlechtlichen Ungleichheit ist, wird sie weiterhin als private Angelegenheit und nicht als öffentliches Feld betrachtet, das einer staatlichen Lösung und Verantwortung bedarf. Anstatt Sorge und die Verantwortung für Sorge zu kollektivieren, wird sie vereinzelt. Die Last, (immer prekärere) Lebensbedingungen zu bewältigen, wird privatisiert und auf das Individuum bzw. die Kleinfamilie verlagert, anstatt öffentliche Strukturen dafür zu schaffen. Es gibt in der Region kaum institutionalisierte Pflegesysteme oder integrierte Politikprogramme, die sich dem Thema Sorge ganzheitlich widmen und Lösungen anbieten, die die Ungleichheit systematisch angehen. Unter 400 pandemiebedingten Unterstützungsmaßnahmen der lateinamerikanischen Regierungen bis Oktober 2020, die die uruguayische Soziologin Karina Batthyány analysiert hat, haben nur die wenigsten eine Geschlechterperspektive mitgedacht - etwa bezüglich der Folgen der Lockdowns hinsichtlich geschlechtsspezifischer und häuslicher Gewalt. Öffentliche Maßnahmen, die die zentrale Frage der zusätzlichen Sorgelast für Frauen adressiert hätten, waren praktisch abwesend (vgl. Batthyány 2021a, 2021: 51:30).

Zudem treffen gleiche Maßnahmen für alle auf die absolute Ungleichheit der Haushalte und auf eine innerhalb der Haushalte ungleiche Arbeitsteilung zwischen Geschlechtern. Einerseits ist die Sorgelast in Haushalten mit geringem Einkommen größer - mehr Mitglieder in einem Haushalt bedeuten auch mehr zu betreuende oder zu pflegende Menschen. Andererseits können reichere Familien ihre Sorgeverpflichtungen aufgrund ihrer finanziellen Möglichkeiten an Dritte, meist informell angestellte Hausarbeiter*innen auslagern und haben in der Regel einen besseren Zugang zu professioneller Pflege. 
Unbezablte Arbeit: das „unsichtbare Rückgrat“ der kapitalistischen Gesellschaft

Die Direktion für Wirtschaft, Gleichstellung und Gender im argentinischen Wirtschaftsministerium hat eine Studie veröffentlicht, die den monetären Wert der unbezahlten Sorge- und Hausarbeit in Argentinien für das Jahr 2020 bemisst (vgl. D'Alessandro et al. 2021). Ziel der Studie ist es, den Wert eines Wirtschaftssektors zu quantifizieren, der grundlegend für alle sozialen, wirtschaftlichen und produktiven Aktivitäten ist. Die Ergebnisse zeigen, dass unbezahlte Sorgearbeit bei einer Vergütung mit Durchschnittslohn ${ }^{3}$ 15,9 Prozent des argentinischen BIP ausmachen würde (entsprechend 67,438 Millionen US-Dollar) und damit den größten Wirtschaftssektor des Landes darstellt. Zu 75,7 Prozent wird diese Arbeit von Frauen getätigt, was 96 Millionen Stunden unbezahlter Arbeit im untersuchten Jahr entspricht. Der Umfang unbezahlter Arbeit ist in der Pandemie um weitere 5,9 Prozent angestiegen und macht für das Jahr 2020 21,8 Prozent des BIP aus. Diese Berechnungen belegen die strategische Bedeutung von Sorgearbeit. Trotzdem bleibt sie sozial und wirtschaftlich unsichtbar.

Die unbezahlte Arbeit stellt also das „unsichtbare Rückgrat“ (D’Alessandro et al. 2021) der kapitalistischen Gesellschaft dar. Sie ermöglicht Menschen, sich zu ernähren, ihre Sorgebedürfnisse zu befriedigen, in wohnbaren Bedingungen zu leben, alltägliche Aktivitäten durchzuführen, sich zu ernähren, arbeiten zu gehen, zu studieren. In Argentinien machen neun von zehn Frauen diese Arbeit an 6,4 Stunden pro Tag. Dies entspricht dem regionalen Durchschnitt, in dem Frauen drei Mal so viel Zeit wie Männer auf unbezahlte Sorgearbeit verwenden. In anderen Worten, so die Studie, tragen Frauen drei Mal mehr zum relevantesten Sektor der argentinischen Volkswirtschaft bei als Männer. Investitionen in Sorgearbeit sind nicht nur nötig, um berufliche Chancen für Frauen und ihre Lebensqualität $\mathrm{zu}$ verbessern, sondern auch um Sorge zu professionalisieren und sozial aufzuwerten.

Nie war der Druck auf eine gesellschaftliche Umstrukturierung und Neuorganisation der Sorgearbeit so groß wie heute, dennoch hat es auch während der Krise nicht die notwendige politische Anerkennung gegeben. Die Regierungen haben mittels Transferleistungen versucht, negative Aus-

3 Der für die Studie errechnete Durchschnittslohn für bezahlte Hausarbeit im vierten Quartal 2019 betrug 86,3 Argentinische Pesos (derzeit etwa 1,30€). Ein konservativ errechneter Wert, der darauf schließen lässt, dass der errechnete Gesamtwert des Sektors niedriger ausfällt (vgl. D’Alessandro et al. 2021: Methodology). 
wirkungen von Lockdowns und Ausgangssperren auf die Sorgelast abzumildern. Das hilft temporär, schafft aber keine langfristigen Lösungen. Anstatt, wie von unterschiedlichen Stellen gefordert, Sorge mit einer generellen Politik als Recht anzuerkennen und allen die Möglichkeit für außerfamiliäre Sorge zu ermöglichen, greifen die lateinamerikanischen Regierungen nur mit einzelnen Unterstützungsleistungen ein, die wiederum verbunden sind mit bestimmten Familienmodellen oder nur zugänglich für formal Beschäftigte. Eine umfassende Sorgepolitik sollte verschiedene Leistungen vereinen. Hierzu gehören u.a. gleichberechtigte Elternzeiten, flexible Teilzeitvereinbarungen, Zugang zu Betreuungsangeboten, die Professionalisierung der Pflege, die Verbesserung der Arbeitsbedingungen für Pflegende und die Förderung kultureller Veränderungen, die es ermöglichen, die Verknüpfung von Sorge und Geschlecht aufzubrechen und klarzumachen, dass Sorge eine intergenerationale Aufgabe aller ist (vgl. Batthyány 2020a: 54:53-56:00). Hierzu gehört aber auch ein „feministisches Grundeinkommen“, ein Lohn für Haus- und Sorgearbeit, der allen ermöglicht das (Über-)Leben im Alltag ohne permanente Krise zu organisieren.

Nicht nur das argentinische Wirtschaftsministerium, auch internationale Organisationen und akademische Studien fordern eine Neustrukturierung der Sorgearbeit, eine Anerkennung der reproduktiven Tätigkeiten, eine Umverteilung der Sorgelast und eine verbesserte Infrastruktur für Sorge (vgl. u.a. CEPAL 2021a: 206ff.). In einer notwendigen Neuorganisation der Arbeitsteilung muss das Kollektive eine größere Rolle spielen. Das Bewusstsein dafür, in welchem Grad die Welt von Sorge abhängig ist, sollte sich in der Verteilung der Ressourcen für diesen systemrelevanten Bereich widerspiegeln.

\section{„Systemrelevante“, aber prekäre Arbeit: Feminisierung der Armut}

Die meisten in der Krise als „systemrelevant" definierten Arbeiten sind feminisiert: Frauen stellen die Mehrheit der Pfleger*innen, des Krankenhauspersonals ${ }^{4}$, der Reinigungskräfte, der Lehrer*innen ${ }^{5}$, der Angestellten in der Lebensmittelindustrie, der Kassierer*innen im Supermarkt. Und sie

4 72,8 Prozent der Beschäftigten im Gesundheitssektor sind Frauen (vgl. Naciones Unidas 2020: 13).

5 70,4 Prozent der Arbeitsplätze im Bildungsbereich war im Jahr 2019 in der Region von Frauen besetzt (vgl. CEPAL 2021a: 210). 
stellen die Mehrheit der prekär Beschäftigten (vgl. CEPAL 2021: 4,11), die in der Krise zuerst ihre Arbeit verloren haben, ohne jegliche soziale Absicherung. 78 Prozent der berufstätigen Frauen in Lateinamerika sind im informellen Arbeitsmarkt und Dienstleistungssektor beschäftigt (vgl. Naciones Unidas 2020: 11). Informelle oder prekäre Beschäftigung bedeutet niedrige Löhne, hohe Instabilität, keine Arbeitssicherheit, Krankenversicherung oder Rentenvorsorge und keinen Schutz vor Arbeitslosigkeit. In der Corona-Krise waren es vor allem die Jobs in der ,informellen“ Wirtschaft, die zuerst weggefallen sind, da sie Präsenz erfordern und keine Infrastruktur für „Homeoffice“ ermöglichen, wie z.B. die Arbeit im Straßenverkauf oder als bezahlte Hausarbeiter*innen. 11,4 Prozent der beschäftigten Frauen in der Region arbeiten als Hausarbeiter*innen, 91,5 Prozent der etwa 13 Millionen Hausarbeiter"innen sind Frauen und mindestens 76 Prozent davon sind informell angestellt (vgl. CEPAL 2020: 3; 2021a: 211). Auch in anderen Wirtschaftszweigen, die besonders stark vom Lockdown betroffen waren ${ }^{6}$, so z.B. in Gastronomie, Hotelgewerbe, Einzelhandel und Tourismus sind Frauen überrepräsentiert und stellen etwa 60 Prozent der Beschäftigten (vgl. Naciones Unidas 2020: 10).

Auch aus diesen Gründen gehen wirtschaftliche Krisen mit der Feminisierung von Armut einher. Es sind oft Frauen, die die höchste Last der Veränderungen im Alltagsleben tragen und zusätzliche Sorgetätigkeiten übernehmen müssen. Armut und Geschlecht überlagern sich aufgrund von patriarchaler und geschlechtsspezifischer Arbeitsteilung. ${ }^{7}$

Fortschritte hinsichtlich der Eingliederung von Frauen in den formalen Arbeitsmarkt aus den letzten Jahrzehnten wurden durch die Pandemie und die pandemiebedingten Maßnahmen zunichtegemacht. Mit einer Neuverteilung der Verantwortung für die Sorgearbeit waren die meist neoliberalen Beschäftigungspolitiken der vergangenen Jahre nicht einhergegangen. Eine umgekehrte Eingliederung von Männern (oder Unternehmen!) in die Verteilung der Haus- und Sorgearbeit hat nie stattgefunden,

6 Die ILO verzeichnet für acht Länder der Region, in denen sie vergleichbare Daten hat, einen Rückgang von 17,6 Prozent im Hotelgewerbe und 12 Prozent für Einzelhandel. In Chile und Costa Rica hat ein Fünftel der Angestellten in der Hotelerie im ersten Quartal 2020 seine Arbeit verloren, in Peru etwa drei von zehn Angestellten im Einzelhandel (vgl. ILO 2021: 48).

7 Bereits vor der Pandemie waren Frauen in Lateinamerika stärker von Armut betroffen als Männer: im Jahr 2018 kamen auf 100 Männer in der Region 113 Frauen (vgl. Naciones Unidas 2020: 13). Diese Ungleichheiten vertiefen sich in der Krise, vor allem in den unteren Einkommensklassen und in Haushalten, in denen Frauen Hauptverdiener*innen sind. 
was bereits vor der Pandemie einen doppelten Arbeitstag für die meisten Frauen zur Folge hatte. Die CEPAL schätzt nun, dass die Pandemie bezüglich der Beteiligung von Frauen am Arbeitsmarkt einen Rückfall auf das Niveau von vor zehn Jahren auslösen wird (CEPAL 2021: 2). Dieser Trend wird langfristige Auswirkungen haben und die Rückkehr von Frauen in die Erwerbstätigkeit ist laut Aussagen der ILO in starkem Maße von der Bereitstellung von öffentlichen Sorgesystemen abhängig (OIT 2020: 11, $37)$.

\section{Schulden machen, um den Alltag zu zablen}

Laut einer Studie des UN-Entwicklungsprogramms (UNDP) für Chile mussten sich 40 Prozent der Haushalte während der Pandemie neu verschulden und zudem ihre Ausgaben, die in Gesundheit, Medikamente und Ernährung fließen, kürzen (UNDP 2020). Die feministischen Theoretikerinnen Veronica Gago und Silvia Federici argumentieren (vgl. u.a. Gago 2020: 16:08-26:58, Federici 2020: 01:25:45-01:29:44), dass sich die Privatverschuldung von Haushalten in der Krise noch verstärkt und zu einem Mechanismus geworden ist, um soziale Reproduktion überhaupt erst möglich zu machen. Das bedeutet Menschen müssen sich notwendigerweise verschulden, um ganz grundlegende Tätigkeiten des Alltags - das Überleben - aufrechtzuerhalten, da die Einnahmen (Lohn, Ersatzleistungen, Sozialhilfen) nicht ausreichen, um die laufenden Kosten für Wohnen, Ernährung, Gesundheit oder z.B. auch Datenvolumen für die Handys zu tilgen.

Die private Verschuldung breitet sich dort aus, wo die Menschen am meisten unter der Krise leiden, wobei Frauen in der Krise aufgrund ihrer Zahlungsmoral und ihrer Netzwerke als Ziel der Kreditgebenden explizit angesprochen werden. Gago spricht hier vom finanziellen Exktraktivismus und der Verschuldung als ein „Dispositiv der Kolonialisierung der sozialen Reproduktion" (Gago 2020: 24:30), da sie in die grundlegenden und notwendigen Aktivitäten der alltäglichen Reproduktion eingedrungen sind: Schulden machen, um den Alltag zu zahlen. Verschuldung wird zum neuen Mechanismus der Ausbeutung in den prekarisierten Teilen der Gesellschaft. Privatverschuldung wird als individuelle „Lösung“ aus der Krise präsentiert, ist aber verbunden mit Privatisierung, Austeritätspolitiken und immer prekäreren Lohnpolitiken, also natürlicherweise Folge einer makroökonomischen Wirtschaftspolitik und nicht - wie es oft dargestellt und empfunden wird - des privaten Versagens oder schlechten Haushaltens. Verschuldung ist, so die Theoretikerinnen, eine Form der Disziplinierung 
und Kontrolle von Frauen in der Zukunft, ein Gehorsamsversprechen, was verschuldete Menschen dazu zwingt, immer noch prekärere Ausbeutungsverhältnisse einzugehen (vgl. Federici 2020: 01:27:54). Alternative Politiken der Entschuldung der privaten Haushalte sind dringend notwendig, um der Verschuldungsspirale zu entgehen.

\section{Kollektive Infrastruktur von unten: Alternativen in der Krise}

Die Krise hat die Notwendigkeit von kollektiven Strategien und Netzwerken deutlich gemacht, die während des Lockdowns in Abwesenheit des Staates Grundbedürfnisse erfüllt haben. In diesen Netzwerken ist als Antwort auf die Krise eine kollektive Infrastruktur von unten entstanden, in der Aufgaben übernommen wurden, die damit zu tun haben, die soziale Reproduktion zu organisieren und die Krise zu managen. Durch solidarische Beziehungen haben vor allem Frauen und Queers die fehlenden Antworten des Staates mit autonomen Aktionen ersetzt, haben wirtschaftliche Überlebensstrategien er- und gefunden, um sich, ihre Familien und Netzwerke zu versorgen. Wie viele beispielhafte Beschreibungen der Krise aus den marginalisierten Stadtvierteln Lateinamerikas schildern (vgl. Baron et al. 2020), sind diese neu entstandenen oder aufgelebten Netzwerke mehr als nur Überlebensstrategie. Durch kreative Prozesse ist ein Raum geschaffen worden, um zu diskutieren, Gemeinschaft zu leben, politische Strategien zu entwickeln und "zärtliche Netzwerke zum Überleben“ (Colectivo Mapeos Feministas 2021: 7) aufzubauen. Die Notwendigkeit zusammenzuhalten, Ressourcen und Kräfte zu kollektivieren, gegenseitig Unterstützung anzubieten, ist die Antwort der Basis auf die Krise. Gemeinschaft dient in der „Schlacht Kapital vs. Leben“ sozusagen als Widerstand, gedacht als Ausdruck selbstorganisierter Formen des Aufbaus von sozialen und wirtschaftlichen Beziehungen, die auf Anerkennung und Wertschätzung von gegenseitiger Abhängigkeit basieren. Für die grundlegendsten Bedürfnisse der Gesellschaft finden sich in der Gemeinschaft kollektive Lösungen, die Wirtschaft und Gesellschaft solidarisch gestalten und die materiellen Voraussetzungen für ein Leben in Würde, ein „lohnenswertes Leben" schaffen (vgl. Colectiva XXK 2021: 37f.). Die reproduktiven Tätigkeiten bleiben, wie Verónica Gago erklärt, nicht nur auf die Haushalte, das Zuhause, das Innere und das Private beschränkt, sondern das „Häusliche“ hat sich auf die Gemeinschaft, auf die Straße und die Viertel erweitert (Gago 2020: 3:30). Sie finden auch in Kiezinitiativen, Stadtteilorganisierungen, gemeinschaftlichen Aktivitäten, Räumen und Netzwerken statt. In der Versorgungsstruktur für Lebensmittel durch kleine Produzent*innen 
und Kooperativen aufgrund von Lieferengpässen, beim Aufräumen und Saubermachen der Viertel, wo die Müllabfuhr nicht hinkommt, dem Organisieren der Nachbarschaftshilfe und Suppenküchen, die während des Lockdowns aus dem Boden geschossen sind und in Argentinien zeitweise 11 Millionen Menschen mit Essen versorgt haben. Essen kochen, aber auch Probleme lösen und über den Umgang mit neuen Ansteckungsfällen in der Nachbarschaft informieren, gehörten zu den Aufgaben dieser Überlebensnetzwerke, ebenso wie Einkäufe und Medikamente organisieren und nach älteren Nachbar*innen zu sehen. Es wurden feministische Notfallpläne, Leitlinien und Warnsysteme für den Umgang mit geschlechtsspezifischer Gewalt entwickelt und gemeinschaftliche und plurinationale Sorgesysteme entworfen (vgl. Coordinadora 8M 2021: 2ff., Manzi 2020). Auf viele verschiedene Weisen haben sich Leute zusammengefunden und organisiert, während die Hilfe des Staats oftmals ausgeblieben ist.

\section{„Ein Riese auf wackeligen Füßen“: Lektionen aus der Krise}

Auch wenn ein Ende der Krise vor allem für die Länder Lateinamerikas und der Karibik noch lange nicht in Sicht ist, werden vielerorts Auswege aus der Krise und Post-Covid Szenarien diskutiert. Lektionen aus der Krise sollten auch nach der Pandemie gelten: ein Zurück in die vermeintliche „Normalität“ ist aus feministischer Perspektive nicht möglich. Der zurückersehnte Normalzustand wird getragen von einem System, das Ungleichheit produziert und diese normalisiert als wäre sie ein natürliches, kein politisches, Phänomen (vgl. Gomez Alcorta 2020: 3). Es ist deutlich geworden, dass die als fortschrittlich gepriesene, globalisierte Gesellschafts- und Wirtschaftsordnung stark krisenanfällig ist. Das kapitalistische System ist nicht in der Lage, Antworten auf globale Krisen zu geben und Leben ausreichend zu schützen. Es ist verwundbar. Es ist zerbrechlich. Ein „Riese auf wackeligen Füßen“, wie die spanische Wirtschaftswissenschaftlerin Amaia Orozco es formuliert (Orozco 2021: 07:57). Zu wissen, dass das System nicht „normal“ ist, dass die Prekarität des Lebens politisch ist, bedeutet eine prinzipielle Veränderung ist möglich. Was die Krise deutlich zeigt, ist, dass sich viele der bestehenden und verschärften Ungleichheiten an der Frage nach Sorge aufzeigen lassen und die Reorganisation der Arbeit und Sorgearbeit als gesellschaftliche Transformationsstrategie ernst genommen werden muss - nicht nur in Lateinamerika, sondern global. Klar ist dabei geworden, dass eine Veränderung der sozialen Reproduktion des Lebens hin zu kollektiven Strukturen notwendig ist: dass Sorgearbeit anerkannt und entlohnt, heraus aus der Isolation der privaten Haushalte geholt und 
vergemeinschaftlicht werden muss. Reichtum muss dazu anders verteilt, in den „Dienst des Lebens“ gestellt werden, wie Silvia Federici sagt. Reichtum sollte in Häuser und Gesundheit investiert werden, und darein, es allen möglich zu machen das alltägliche Leben zu reproduzieren ohne Ungleichheit, ohne Ausbeutung und ohne Elend (vgl. Federici 2020: 29:3631:36).

All dem liegt die Idee zugrunde, statt dem patriarchalen, globalen Kapitalismus das Leben, die Solidarität und die Sorge ins Zentrum zu holen und darüber Alternativen für eine Welt in der Krise zu finden. Das bedeutet kollektive Antworten einer sozialen, solidarischen, feministischen Ökonomie auf die Krise der sozialen Reproduktion. In diesen Prozessen, wie sie die Corona-Krise nun vielfach zu Tage gebracht hat, entsteht mehr als Sorge. Es entstehen Räume, in denen die heutige Art zu leben problematisiert wird. Es entstehen neue Formen des Widerstands, die die kapitalistische Produktionsweise infrage stellen.

\section{Literatur- und Quellenverzeichnis}

Barón, Camila; Bascuas, Maisa; Bustos, Ana Julia; Roco, Josefina; Rosales, Josefina; Roggi, Magdalena (2020): Destapar la Crisis: Guardianas de la Comunidad, in: Lado A+B, [Podcast] [https://spoti.fi/3hnFp7u], letzter Aufruf 05.09.2021.

Batthyány, Karina (2020, 28.04): Las desigualdades de géneros y cuidados ante la pandemia, in: CLACSOTV, [Video] [http://youtu.be/89u2C5WYLws], letzter Aufruf 05.09.2021.

Batthyány, Karina (2020a, 29.04.): Conferencia Pandemia Global, in: Vicerrectoría De Desarrollo Humano, [Video] [https://bit.ly/2YQhuah], letzter Aufruf 05.09.2021.

Batthyány, Karina (2021, 15.03): A un año de la pandemia ¿Qué ocurre con los cuidados en clave de género?, in: CLACSO TV, [Video] [http://youtu.be/vn6EIW 0Phis], letzter Aufruf 05.09.2021.

Batthyány, Karina (2021a, 03.05.): Esta pandemia llega a América Latina y el Caribe en una alta desigualdad, vulnerabilidad y debilitamiento social, in: CLACSO, [Online] [https://bit.ly/3nvlkzN], letzter Aufruf 05.09.2021.

CEPAL (2020): La pandemia del COVID-19 profundiza la crisis de los cuidados en América Latina y el Caribe [PDF] [https://bit.ly/3z4SFE2], letzter Aufruf 05.09.2021.

CEPAL (2021a): Panorama Social de América Latina 2020, Santiago de Chile: CEPAL.

CEPAL (2021): La autonomía económica de las mujeres en la recuperación sostenible y con igualdad, [PDF] [https://bit.ly/3k7zcyh], letzter Aufruf 05.09.2021. 
Colectiva XXK \& Sempreviva Organização Feminista (2021): Vereint und rebellisch: Auf Entdeckung der Territorien der feministischen Ökonomie, [PDF] [https://bit.ly/31 fpfOx], letzter Aufruf 05.09.2021.

Colectivo Mapeos Feministas (2021): Destapar la Crisis: Trabajos de Cuidados en Tiempos de Coronavirus, in: Dossier $n^{\circ} 38$, Instituto Tricontinental de Investigación Social, [PDF] [https://bit.ly/3k5fjrC], letzter Aufruf 05.09.2021.

Coordinadora 8M (2021): Juntas avanzamos hasta dignificar los trabajos de cuidado, in: La Primera vom 08.05.2021, [PDF] [https://bit.ly/3lhsjtq], letzter Aufruf 05.09.2021.

D’Alessandro, Mercedes; O’Donell, Victoria; Prieto, Sol; Tundis, Florencia; Zanino, Carolina (2021): The Value of Care: A Strategic Economic Sector: A Measurement of Unpaid Care and Domestic Work in the Argentine GDP, [PDF] [https://bit.l y/3EbcywF], letzter Aufruf 05.09.2021.

Federici, Silvia (2020, 29.07.): Desafíos feministas en tiempos de pandemia, [Video] [https://youtu.be/unu-xGO8JiQ], letzter Aufruf 05.09.2021.

Gago, Verónica (2020, 20.08.): VIRAL. Proyecciones de una Argentina Post-Pandemia. Entrevista con Verónica Gago, ADiUC. [Video] [https://bit.ly/2YRJHgT], letzter Aufruf 05.09.2021.

Gago, Verónica; Cavallero. Lucía (2020): Deuda, Vivienda y Trabajo: Una Agenda feminista para la Pospandemia, in: Revista Anfibia, [online] [https://bit.ly/3htwd OL], letzter Aufruf 05.09.2021.

Gomez Alcorta, Eli (2020): Prefacio, In: Instituto Tricontinental de Investigación Social, Coronashock y Patriarcado, [PDF] [https://bit.ly/3EaiDcK], letzter Aufruf 05.09.2021.

López-Calva, Luis Felipe (2021): COVID-19 y la riqueza en la cima: más multimillonarios y más ricos en ALC tras la crisis, [online] [https://bit.ly/3nnlicd], letzter Aufruf 05.09.2021.

Manzi, Javiera (2020): Wir haben uns das Leben zu eigen gemacht, in: Lateinamerika Nachrichten 551, Online Ausgabe, [Online] [https://bit.ly/3Ea1kbY/], letzter Aufruf 05.09.2021.

Naciones Unidas (2020): Informe: El impacto del COVID-19 en América Latina y el Caribe, [PDF], [https://bit.ly/394PWjj], letzter Aufruf 05.09.2021

OIT (2020): PANORAMA LABORAL 2020: América Latina y el Caribe, [PDF] [https://bit.ly/3lhE6YJ], letzter Aufruf 05.09.2021.

Orozco, Amaia (2020): Destapar la crisis: Ese gigante de pies de barro, in: Lado A, [Podcast] [https://spoti.fi/2Vzf66o], letzter Aufruf 05.09.2021

Sagot, Montserrat (2020, 20.04.): Estamos viviendo una crisis civilizatoria, in: CLACSOTV, [Video] [https://youtu.be/f9nr-BdZo5w], letzter Aufruf 05.09.2021.

UNDP (2020): Impactos socioeconómicos de la pandemia en los hogares de Chile, [PDF] [https://bit.ly/3hquslu], letzter Aufruf 05.09.2021.

Von Redecker, Eva (2020): Revolution für das Leben: Philosophie der neuen Protestformen, 2. Aufl., Frankfurt a.M.: S. Fischer Verlag. 
